\title{
Effects of Helicobacter pylori on the expression levels of GATA-3 and connexin 32 and the GJIC function in gastric epithelial cells and their association by promoter analysis
}

\author{
LIHUA HUANG ${ }^{1}$, YINJIE GUO ${ }^{2}$, DAN CAO $^{2}$, XIAOMING LIU ${ }^{2}$, LINFANG ZHANG ${ }^{2}$, \\ $\mathrm{KE} \mathrm{CAO}^{3}$, TINGZI HU ${ }^{2}$, YONG $\mathrm{QI}^{4}$ and CANXIA XU ${ }^{2,5}$ \\ ${ }^{1}$ Center for Medical Experiments; Departments of ${ }^{2}$ Gastroenterology and ${ }^{3}$ Oncology; ${ }^{4}$ Clinical Laboratory; \\ ${ }^{5}$ Hunan Key Laboratory of Nonresolving Inflammation and Cancer, Third Xiangya Hospital of \\ Central South University, Changsha, Hunan 410013, P.R. China
}

Received July 6, 2016; Accepted March 29, 2018

DOI: $10.3892 / \mathrm{ol} .2018 .8796$

\begin{abstract}
The present study aimed to explore the effects of Helicobacter pylori (H. pylori) infection on the expression of transcription factor GATA binding protein 3 (GATA-3) and connexin 32 (Cx32) in cultured gastric mucosa cells, and their association with each other. GES-1 cells were co-cultured with East Asian type cytotoxin-associated gene $\mathrm{A}^{+} H$. pylori in the $H$. pylori group, and without $H$. pylori culture in the control group. Additionally, Mongolian gerbils were gavaged with $H$. pylori, and later the gastric antrum tissues were collected. The GATA-3 and Cx32 mRNA and protein expression levels were detected by a reverse transcription-quantitative polymerase chain reaction and western blot analysis, respectively. The scratch labeling fluorescent dye tracer (SLDT) technique was used to detect the gap junctional intercellular communication (GJIC) function. GATA-3 small interfering RNA (siRNA) was transfected into BGC823 cells and its effect on Cx32 expression levels was detected. The impact of GATA-3 on Cx32 promoter transcriptional activity was detected using a dual luciferase reporter assay. The results revealed that $H$. pylori infection increased GATA-3 expression and decreased Cx32 expression in GES-1 cells and in animal gastric tissues compared with their respective controls, whilst in BGC823 cells, GATA-3 siRNA increased Cx32 expression compared
\end{abstract}

Correspondence to: Professor Canxia $\mathrm{Xu}$, Department of Gastroenterology, Third Xiangya Hospital of Central South University, 138 Tongzipo Road, Changsha, Hunan 410013, P.R. China E-mail: xucanxia2000@163.com

Abbreviations: CagA, cytotoxin-associated gene A; GATA-3, GATA binding protein 3; Cx32, connexin 32; GJIC, gap junction intercellular communication; NAG, non-atrophic gastritis; CAG, chronic atrophic gastritis; IM, intestinal metaplasia; DYS, dysplasia; NGM, normal gastric mucosa; GC, gastric cancer

Key words: Helicobacter pylori, connexin 32, gap junctional intercellular communication, gene promoter with the control. In the SLDT experiment of GES-1 cells with H. pylori infection, the fluorescent dye was primarily limited to a single cell row close to the scratch, and only a limited amount of dye passing to the second cell row, indicating that the GJIC function was substantially reduced or absent compared with the control group, where the fluorescence dye transferred to the neighboring cells of 3-4 rows, indicating a stronger GJIC function comparatively. GATA-3 inhibited the expression of the luciferase reporter gene, compared with the controls, suggesting that GATA-3 inhibited the expression of Cx32 by binding to $C x 32$ promoter sites. These results indicated that H. pylori-increased GATA-3 expression, which downregulated Cx32 expression, may serve an important function in gastric carcinogenesis, and GATA-3 siRNA may serve a function in the prevention and treatment of gastric cancer.

\section{Introduction}

The incidence and mortality rates of gastric cancer (GC), a common cancer threatening to human health, are the second and third highest, respectively, in China in 2015 (1). Gastric carcinogenesis is a multi-gene, multi-step complex process (2). It is generally considered that Helicobacter pylori (H. pylori) may serve a substantial function in the gastric 'inflammation-cancer chain' composed of chronic non-atrophic gastritis (NAG), chronic atrophic gastritis (CAG), intestinal metaplasia (IM), dysplasia (DYS) and GC (3). The risk of GC in individuals infected with $H$. pylori was 3-6 times higher compared with uninfected individuals (4), and animal experiments confirmed that $H$. pylori may induce Mongolian gerbil gastric adenocarcinoma $(5,6)$. The underlying mechanisms of $H$. pylori resulting in GC may have a plurality of pathways, one of which may be the loss of the connection between the cells. Gap junctions are the ubiquitous connection between animal cells, mediating the gap junction intercellular communication (GJIC) to participate in the exchange of materials between cells, metabolic coupling and electrical transmitting, performing the exchange of information and energy substances and serving a notable regulatory function in physiological processes including cell metabolism, homeostasis, proliferation and differentiation (7). 
Connexin $32(\mathrm{Cx} 32)$ is the main member of gastric epithelial cell gap junctions. In the development of $\mathrm{GC}$, the expression of Cx32 demonstrates a gradual downward trend from normal gastric mucosa (NGM) to precancerous lesions to GC (8). The decrease of Cx32 expression is an early molecular event in the process of GC. One previous study revealed that the lowered expression of $\mathrm{Cx} 32$ in precancerous lesions and GC is closely associated with $H$. pylori infection, and the co-culturing of gastric epithelial cells and H. pylori for 24 or 48 h significantly decreased the expression of Cx32 (9), and the eradication of $H$. pylori may increase the Cx32 expression in gastric precancerous lesions (10). In addition, clinical and in vitro experimental studies have confirmed that $H$. pylori may significantly reduce gastric epithelial cell GJIC function $(11,12)$. These studies indicated that $H$. pylori infection may reduce the expression of $\mathrm{Cx} 32$ in gastric epithelial cells, thereby inhibiting GJIC function, which may be the mechanism underlying $H$. pylori-induced GC, but the specific mechanism of $H$. pylori that results in $\mathrm{Cx} 32$ decline in gastric epithelial cells remains unclear.

One previous study performed a high-throughput transcription factor screening in NGM tissue without $H$. pylori infection and in gastric mucosa tissues at five different stages of the gastric 'inflammation-cancer chain' with $H$. pylori infection $(\mathrm{NAG} \rightarrow \mathrm{CAG} \rightarrow \mathrm{IM} \rightarrow \mathrm{DYS} \rightarrow \mathrm{GC}$ ), and revealed that the GATA binding protein 3 (GATA-3) expression was low in normal mucosa, comparatively increased significantly in NAG and precancerous (CAG, IM and DYS) mucosa and highest in GC (13). Bioinformatics software analysis revealed that there are multiple GATA-3 binding sites in the promoter regions of Cx32 genes. Therefore, it was hypothesized that $H$. pylori infection may downregulate $\mathrm{Cx} 32$ expression by upregulating GATA-3 expression in gastric epithelial cells, thus serving an important function in the gastric carcinogenesis caused by H. pylori infection.

The present study intended to co-culture gastric epithelial cells with $H$. pylori and collect the gastric antrum tissues from $H$. pylori-gavaged animals in order to observe the effects of H. pylori on GATA-3, Cx32 expression and GJIC function, to silence GATA-3 by small interfering RNA (siRNA) transfection to observe its effects on Cx32 expression and to conduct dual luciferase reporter experiments by constructing a Cx32 promoter vector. These experiments attempted to elucidate whether $H$. pylori infection results in Cx32 downregulation by upregulating GATA-3, and whether this is associated with GC.

\section{Materials and methods}

Co-culture of gastric epithelial cells with H. pylori. H. pylori strains were isolated from five patients (4 males and 1 female; mean age, 55 years; age range, 40-70 years) diagnosed with GC subsequent to endoscopic and pathological examinations in July 1-31st, 2012 at the Third Xiangya Hospital of Central South University (Changsha, China), cultured and identified as $H$.pylori by the bacteria colony morphology, and activity assays of its enzymes, including urease (producing carbon dioxide and ammonia), catalase (producing oxygen) and oxidase assays, and identified as East Asian type cytotoxin-associated gene $\mathrm{A}(\mathrm{CagA})^{+} H$. pylori strains by CagA gene sequencing. Written informed consent was obtained from all patients prior to the study. Normal human gastric epithelial cell line GES-1 was purchased from Shanghai Bogoo Biotechnology Co., Ltd. (Shanghai, China) and the human GC cell line BGC823 was gifted from the Cancer Research Institute, Central South University (Changsha, China).

Cells of logarithmic growth phase were digested with trypsin to prepare a single cell suspension, counted using a hemocytometer, seeded in $10^{5} /$ well in 6 -well plates, and cultured at $37^{\circ} \mathrm{C}$ and $5 \% \mathrm{CO}_{2}$. Once the cells adhered firmly by observation under a light microscope, Dulbecco's modified Eagle's medium (DMEM) without fetal bovine serum (Thermo Fisher Scientific, Inc., Waltham, MA, USA) was used to culture the cells for $24 \mathrm{~h}$ to synchronize the cells at the G0 phase. H. pylori bacteria were collected using sterile phosphate buffered saline (PBS), the concentrations measured using an ultraviolet spectrophotometer $\left(\mathrm{OD}_{660}=10^{8} / \mathrm{ml}\right)$, and $H$. pylori bacteria and GES-1 cells were seeded at a ratio of 50:1 in 6-well plates and co-cultured at $37^{\circ} \mathrm{C}$ for 12 and $24 \mathrm{~h}$.

Collection of gastric tissues from normal and H. pylorigavaged animals. A total of 20 male Mongolian gerbils (aged 6-8 weeks; weight, 51.9-66.8 g) purchased from the Animal Experimental Center of Zhejiang Province (Hangzhou, China), were housed and fed with food and water in individually ventilated cages, with $12 / 12 \mathrm{~h}$ dark/light cycle, at $25^{\circ} \mathrm{C}$ temperature. They were randomly allocated to the control ( 5 animals) and experimental (15 animals) groups. The animal ethics protocol was approved by the Ethics Committee of the Third Xiangya Hospital of Central South University (Changsha, China). After all the gerbils fasted for $24 \mathrm{~h}$, the experimental group were administered $H$. pylori gavage (East-Asian type $\mathrm{CagA}^{+}$from patients with GC, once a day for 7 days total), and the controls were administered saline gavage. After 48 weeks, the gerbils were anaesthetized with chloral hydrate $(400 \mathrm{mg} / \mathrm{kg})$ and a chest and abdominal incision was carefully made. Perfusion was performed using PBS, preventing the heart from beating and resulting in euthanasia. The gastric antrum tissues were collected and stored in liquid nitrogen immediately for further detection of GATA-3 and Cx32 expression.

Western blot analysis. The GES-1 and BGC823 cells were washed with cool PBS for 3 times, lyzed with radioimmunoprecipitation assay lysis buffer (Beyotime Institute of Biotechnology, Shanghai, China) on ice for 15-30 min, transferred to tubes, centrifuged at $12,000 \mathrm{x} \mathrm{g}$ at $4^{\circ} \mathrm{C}$ for $5 \mathrm{~min}$, and the supernatant transferred to a new tube, thus the protein was extracted. The protein concentration was measured by BCA protein assay kit (Beyotime Institute of Biotechnology). The proteins were electrophoresed (20 $\mu \mathrm{g} /$ lane) via SDS-PAGE (10-12.5\% gel) and transferred to polyvinylidene fluoride membranes, washed with Tris-buffered saline and Tween (TBST) for 5 min, blocked in 5\% skimmed-milk in TBST, and hybridized with primary antibodies. The anti-human antibodies, including rabbit anti-Cx32 (cat. no. 10450-1-AP; dilution, 1:800), rabbit anti-GATA3 (cat. no. 10417-1-AP; dilution, 1:800), mouse anti- $\beta$-actin (cat. no. 600008-1; dilution, 1:4,000) and the secondary antibodies (horseradish peroxidase-labeled goat anti-rabbit or anti-mouse antibodies; cat. nos. SA00001-2 and SA00001-1, respectively; dilution, 1:3,000) were purchased from ProteinTech Group, 
Inc. (Chicago, IL, USA). The hybridization for primary antibodies was incubated at $4{ }^{\circ} \mathrm{C}$ overnight, and that for secondary antibodies was at room temperature for $45 \mathrm{~min}$. ECL solutions (Thermo Fisher Scientific, Inc.) were added to the membrane and images of the protein bands were captured and analyzed by the Image Quant 350 Gel Protein Imaging System (version 1.0.2; GE Healthcare) and Image Analysis Software (version 7.0; GE Healthcare).

Reverse transcription-quantitative polymerase chain reaction $(R T-q P C R)$. Total RNA was extracted from cells or tissues by grinding with liquid nitrogen, adding TRIzol ${ }^{\circledR}$ reagent (Invitrogen; Thermo Fisher Scientific, Inc.), followed by chloroform, isopropanol, and $75 \%$ ethanol, each time centrifuging at $12,000 \mathrm{x}$ at $4^{\circ} \mathrm{C}$ for $15 \mathrm{~min}$, and finally drying to be dissolved in $0.1 \%$ diethylpyrocarbonate water (Shanghai Sangon Biotechnology Co., Shanghai, China). RNA was reverse transcribed into cDNA using a SuperScript Reverse Transcription kit (Thermo Fisher Scientific, Inc.). Briefly, Oligo(dT) $)_{15}$ primer was added and heated at $65^{\circ} \mathrm{C}$ for $10 \mathrm{~min}$ and cooling to $4^{\circ} \mathrm{C}$ on ice for $10 \mathrm{~min}$, then the buffer, dNTP mix and reverse transcriptase from the kit were added for reaction at $42^{\circ} \mathrm{C}$ for $1 \mathrm{~h}$, and finally the reaction was inactivated by heating at $70^{\circ} \mathrm{C}$ for $15 \mathrm{~min}$. The RT-qPCR reaction was conducted using the $\mathrm{SYBR}^{\circledR}$-Green Master Mix (Thermo Fisher Scientific, Inc.) in the Realplex qPCR instrument (Eppendorf, Hamburg, Germany) under the following conditions: $95^{\circ} \mathrm{C}$ for $5 \mathrm{~min}$, and then $95^{\circ} \mathrm{C}$ for $15 \mathrm{sec}, 55^{\circ} \mathrm{C}$ for $15 \mathrm{sec}$ and $72^{\circ} \mathrm{C}$ for $30 \mathrm{sec}$, for 40 cycles. Following this, the results were analyzed by the $2^{-\Delta \Delta \mathrm{Cq}}$ method (14). The primers were designed using Primer 5.0 software (Premier Biosoft, Palo Alto, CA, USA) and synthesized from the Shanghai Sangon Biological Engineering Co. The PCR primer sequences for human cells are as follows: GATA-3 forward, 5'-GGAGTGTGTGAACTGTGGGG-3' and reverse, 5'-TTC GCTTGGGCTTAATGAGG-3'; Cx32 forward, 5'-GGATGC TCCGACAGCGTCTC-3' and reverse, 5'-GCCCTCTGCTCC TCTTACCC-3'; $\beta$-actin forward, 5'-AGGGGCCGGACTCGT CATACT-3' and reverse, 5'-GGCGGCACCACCATGTAC CCT-3'. The PCR primer sequences for gerbil gastric tissue were as follows: GATA-3 forward, 5'-CGGCAGGCAGAT GAGAAAG-3' and reverse, 5'-GGGCACATAGGGCGGA TAG-3'; Cx32 forward, 5'-CACCAACAGCACATAGAA AAG-3' and reverse, 5'-GATGACATAGGTCCACCACAG-3'; $\beta$-actin forward, 5'-CCCATCTATGAGGGTTACGC-3' and reverse, 5'-TTTAATGTCACGCACGATTTC-3'.

Detection of GJIC function in GES-1 cells. Scrape-loading and fluorescent dye transfer (SLDT) technique was used to detect the GJIC function in GES-1 cells with or without $H$. pylori infection. Briefly, GES-1 cells were co-cultured in 6 -well plates at $37^{\circ} \mathrm{C}$ with $95 \%$ air and $5 \% \mathrm{CO}_{2}$ to show monolayer cell concurrence under light microscopy (magnification, $\mathrm{x} 40$ ). The medium was removed, and each well of cultured cells was washed with sterile PBS gently for $5 \mathrm{sec}$ at room temperature for 4 times. A total of $1 \mathrm{ml}$ PBS containing $0.5 \%$ Lucifer Yellow (Thermo Fisher Scientific, Inc.), was added, and the cell monolayer was gently scraped with a sharp blade to form a scratch $\sim 50-\mu \mathrm{m}$ wide. Cells were kept still for $3 \mathrm{~min}$, the fluid was discarded, and the well was washed again with sterile PBS gently for $5 \mathrm{sec}$ at room temperature for 4 times to wash away the residual fluorescent dye. Fluorescence and ordinary light images were taken from the same vision field under an inverted fluorescence microscope (magnification, $\mathrm{x} 40$ ) (IX71; Olympus Corporation, Tokyo, Japan). By observing the cells with fluorescent dye next to the scrape line, the transfer distance or the number of cells the dye passed, the functional status of GJIC was determined. A greater number of cells to which the fluorescent dye had spread to was considered to indicate a stronger GJIC function.

Transfection of GATA-3 siRNA into BGC823 cells. BGC823 cells were cultured to $80-90 \%$ confluence, digested with trypsin, carefully drawn off using a pipette into a single cell suspension, and seeded into $100 \mathrm{~mm}$-diameter cell culture dishes $(10 \mathrm{ml} / \mathrm{dish})$ with a cell density of $2 \times 10^{5}$ cells $/ \mathrm{ml}$. The cells were cultured at $37^{\circ} \mathrm{C}$ and $5 \% \mathrm{CO}_{2}$ in an incubator for $24 \mathrm{~h}$, then the DMEM medium (Thermo Fisher Scientific, Inc.) was changed, and when the cells were $80 \%$ confluent, transfection was performed using Lipofectamine ${ }^{\circledR} 2000$ (Invitrogen; Thermo Fisher Scientific, Inc.), according to the manufacturer's protocol. They were divided into three groups: i) No treatment as the control group; ii) transfected with negative control as the NC group; and iii) transfected with $10 \mu \mathrm{M}$ GATA-3 siRNA as the siRNA group. A total of $48 \mathrm{~h}$ after transfection, RNA or proteins were extracted. siRNA was synthesized by Shanghai GenePharma Co., Ltd. (Shanghai, China) with the following sequences: GATA-3 siRNA forward, 5'-CAUCGACGGUCA AGGCAACTT-3' (the first 19 nucleotides corresponding to the GATA-3 coding sequence nucleotides 156-174, and TT for protection) and reverse. 5'-GUUGCCUUGACCGUCGAU GTT-3'; negative control forward, 5'-UUCUCCGAACGUGUC ACGUTT-3' and reverse, 5'-ACGUGACACGUUCGGAGA ATT-3'.

Construction of pYr-PromDetect-Cx32P vector. The fragment of $\mathrm{Cx} 32$ promoter -651 to +84 (transcription start site as +1 , total 735 base pairs) was PCR amplified from human genomic DNA by the following primers with restriction enzyme $B g l \mathrm{II}$ and NheI sites added (underlined respectively): Forward, 5'-GAAGATCTAATGGTTAGCCTTTGCTTTC-3' and reverse, 5'-CTAGCTAGCTATGGTCTCATGTCTGTGC AGG-3'. The PCR reaction was performed using PrimeSTAR-HS DNA polymerase in PCR buffer containing $2.5 \mathrm{mM}$ dNTP (Takara Bio, Inc., Otsu Japan), with the thermocycling condition: $94^{\circ} \mathrm{C}$ for $2 \mathrm{~min}$, then $94^{\circ} \mathrm{C}$ for $30 \mathrm{sec}, 55^{\circ} \mathrm{C}$ for $30 \mathrm{sec}$ and $72^{\circ} \mathrm{C}$ for $90 \mathrm{sec}$, for 30 cycles, followed by $72^{\circ} \mathrm{C}$ for $3 \mathrm{~min}$. The amplified fragment was inserted into the pYr-PromDetect vector (pLuc; Changsha Yingrun Biotechnology Co., Ltd., Changsha, China) and formed pYr-PromDetect-Cx32P vector (pLuc-Cx32P). The sequencing result displayed an exact alignment with the $\mathrm{Cx} 32$ promoter sequence in the National Centre for Biotechnology Information (NCBI; https:// www.ncbi.nlm.nih.gov/pubmed).

Determination of potential GATA-3 binding sites in the Cx32 promoter. The DNA consensus sequence that the GATA-3 transcription factor binds to is 5'-A/T-GATA-A/G-3', thus the consensus sequence in the contrary strand is 5'-T/C-TATC-T/A-3' $(15,16)$. For the aforementioned cloned 
sequence of the $C x 32$ promoter, a search was made for GATA or TATC, which also satisfied the nucleotide requirement at the $5^{\prime}$ and $3^{\prime}$ ends, and the potential binding sites of GATA3 was identified to be -623 to $-618,-514$ to -509 and -239 to -234.

GATA-3 expression vector. The complete coding sequence of GATA-3 was PCR amplified from human cDNA by using the following primers with additional NheI and XhoI digestion sites added (underlined respectively): Forward, 5'-CGCTAG CGATGGAGGTGACGGCGGA-3' and reverse, 5'-GCCTCG AGCTAACCCATGGCGGTGAC-3'. The PCR reaction was performed using PrimeSTAR-HS DNA polymerase in PCR buffer containing $2.5 \mathrm{mM}$ dNTP (Takara Bio, Inc.), with the thermocycling condition: $94^{\circ} \mathrm{C}$ for $2 \mathrm{~min}$, then $94^{\circ} \mathrm{C}$ for $30 \mathrm{sec}$, $55^{\circ} \mathrm{C}$ for $30 \mathrm{sec}$ and $72^{\circ} \mathrm{C}$ for $90 \mathrm{sec}$, for 30 cycles, followed by $72^{\circ} \mathrm{C}$ for $3 \mathrm{~min}$. The amplified fragment was inserted into a GV230 vector (pGv) and formed the pGATA3 vector. The sequencing result was matched against the GATA-3 NCBI sequence.

Dual-luciferase reporter experiment. The luciferase detection kit was purchased from Changsha Yingrun Biotechnology Co., Ltd. and performed according to the manufacturer's protocol. Briefly, GES-1 cells in the logarithmic growth phase and growing in an active condition were seeded in 24-well plates. When the cell density reached $70-80 \%$ in the well, $2 \times 10^{5} \mathrm{GES}-1$ cells were transfected with the plasmids (pYr-PromDetect$\mathrm{Cx} 32 \mathrm{P}$ containing the test promoter sequence $\mathrm{Cx} 32 \mathrm{P}$, or/and pGATA3 expressing the transcription factor GATA3 to bind to the test promoter) by Lipofectamine 2000 (Invitrogen; Thermo Fisher Scientific, Inc.) according to the manufacturer's protocol, and cultured at $37^{\circ} \mathrm{C}$ and $5 \% \mathrm{CO}_{2}$ in an incubator. After $48 \mathrm{~h}$, the cells were washed with 1X PBS (100 $\mu \mathrm{l} / \mathrm{well}$, one time), cleaved by gentle shaking at room temperature for $15 \mathrm{~min}$, and the lysates were transferred to a detection tube. When measuring in the luminometer (PerkinElmer, Inc., Waltham, MA, USA), $100 \mu 1$ Luciferase Assay Reagent-II and stop-Glo reagent were automatically added and the results read using 1-2 sec and 5-10 sec delays.

Using a dual luciferase reporter assay, the $C \times 32$ promoter sequence -651 to +84 (containing TATA box at position -15 and transcription start site) was inserted into the 5' upstream of Renilla luciferase(Rluc) coding sequence in the reporter plasmid, immediately next to the Kozak sequence GCCACCATG, starting the translation of Rluc. Furthermore, the Firefly luciferase (Fluc) coding sequence was subsequent to the promoter sequence derived from cytomegalovirus in the same plasmid producing stable expression following transfection. The ratio of Rluc fluorescence to Fluc fluorescence (R/F ratio) reflected the activity of the inserted promoter sequence.

Statistical analysis. SPSS 19.0 statistical software (IBM Corp., Armonk, NY, USA) and GraphPad Prism 7.00 software (GraphPad Software, Inc., La Jolla, CA, USA) were used for statistical analysis. The data were calculated and presented as mean \pm SD. Measurement data were tested and distributed normally. Statistical significance of the data was assessed by a Student's unpaired t-test between two groups, or one-way analysis of variance followed by the post hoc Bonferroni test
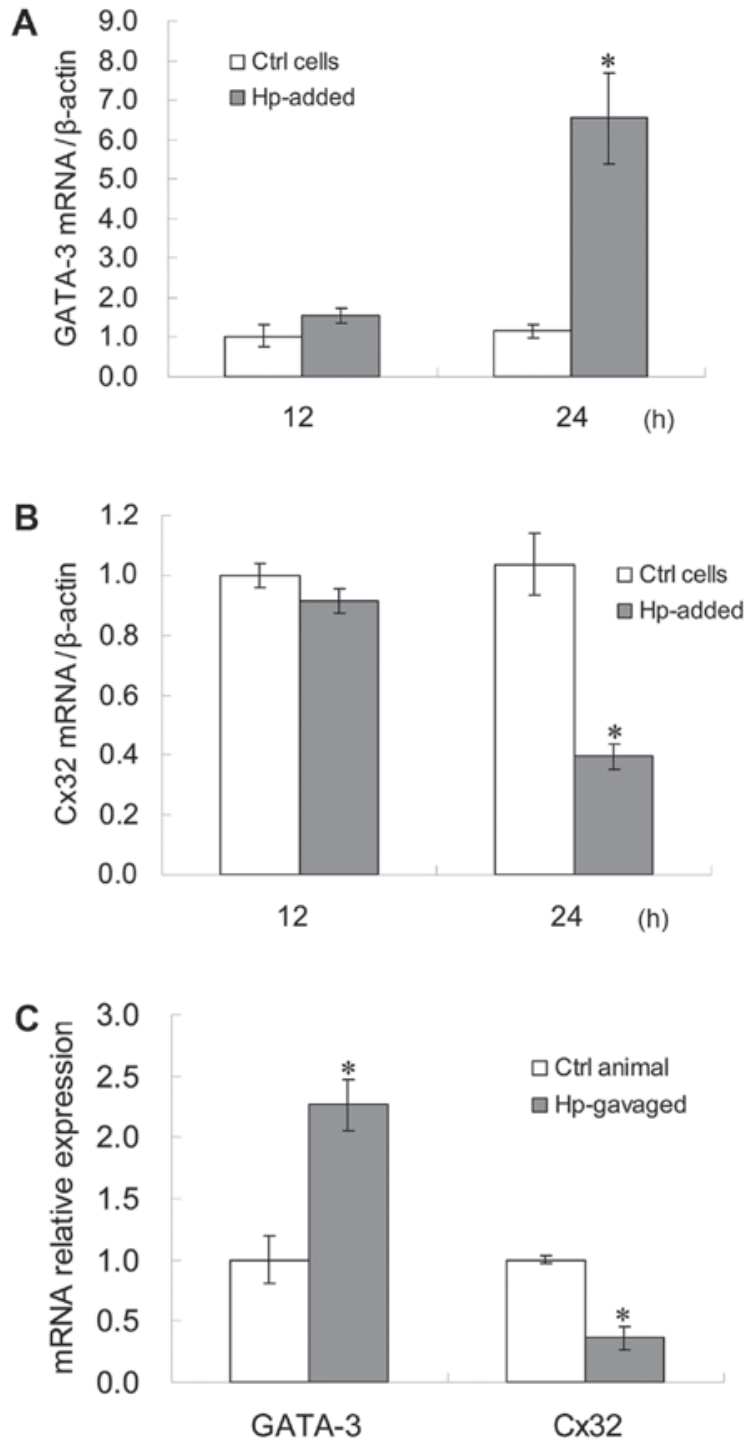

Figure 1. GATA-3 and Cx32 mRNA expression following $H$.pylori infection. (A) GATA-3 mRNA expression levels in GES-1 cells following $H$. pylori infection for 12 and $24 \mathrm{~h}$. (B) Cx32 mRNA expression levels in GES-1 cells following $H$. pylori infection for 12 and $24 \mathrm{~h}$. (C) GATA-3 and Cx32 mRNA expression levels in gerbil gastric antrum tissues in gerbils gavaged with H. pylori. ${ }^{*} \mathrm{P}<0.05$ vs. the control. GATA-3, GATA binding protein 3 ; Cx32, connexin $32 ;$ H. pylori/Hp, Helicobacter pylori; Ctrl, control.

among multiple groups. $\mathrm{P}<0.05$ was considered to indicate a statistically significant difference.

\section{Results}

Changes of GATA-3 and CX32 mRNA expression subsequent to H. pylori infection. GATA-3 mRNA expression levels in GES-1 cells at $24 \mathrm{~h}$ following $H$. pylori infection were significantly higher compared with the control $(\mathrm{P}<0.05$; Fig. 1A), while Cx32 mRNA expression levels in GES-1 cells at $24 \mathrm{~h}$ following $H$. pylori infection were significantly lower compared with the control $(\mathrm{P}<0.05$; Fig. 1B). The correlation between GATA-3 and Cx32 mRNA expression levels in GES- 1 cells at $24 \mathrm{~h}$ after $H$. pylori infection was statistically significant ( $\mathrm{r}=-0.6713 ; \mathrm{P}=0.0477$ ) (data not shown). GATA-3 mRNA expression levels in the gerbil gastric antrum tissues subsequent to $H$. pylori infection were significantly higher 


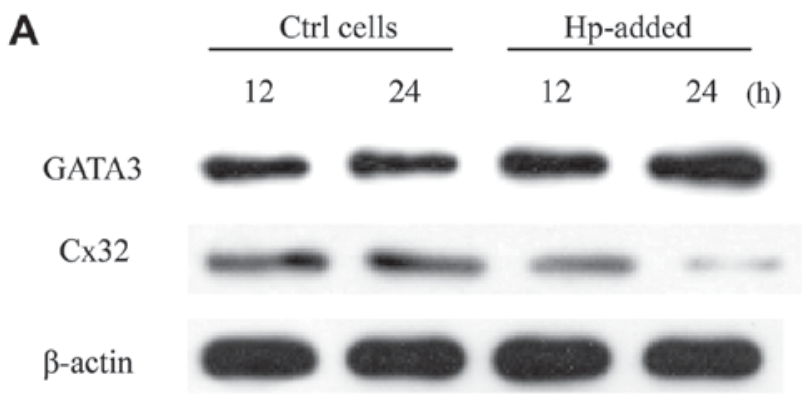

B

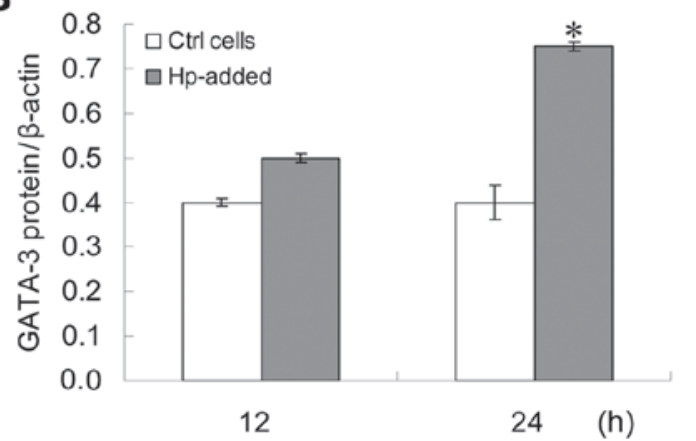

C $\quad 0.4$

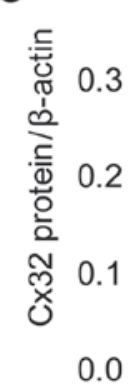

0.0

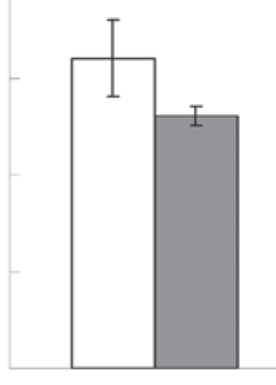

12

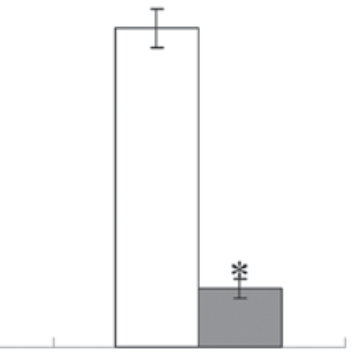

(h)

Figure 2. Protein expression levels of GATA-3 and Cx32 in GES-1 cells subsequent to Helicobacter pylori infection. (A) Bands of western blot analysis subsequent to electrophoresis. (B) GATA-3 protein expression levels in GES-1 cells. (C) Cx32 protein expression levels in GES-1 cells. * $\mathrm{P}<0.05$, compared with control. GATA-3, GATA binding protein 3; Cx32, connexin 32; Hp, Helicobacter pylori; Ctrl, control.

compared with the control, while Cx32 mRNA expression levels in the gerbil gastric antrum tissues subsequent to H.pylori infection were significantly lower compared with the control $(\mathrm{P}<0.05$; Fig. 1C).

Changes of GATA-3 and Cx32 protein expression levels in GES-1 cells subsequent to $H$. pylori infection. GATA-3 protein expression levels in GES-1 cells at $24 \mathrm{~h}$ following $H$. pylori infection were significantly higher compared with the control, while Cx32 protein expression levels in GES-1 cells at $24 \mathrm{~h}$ following $H$. pylori infection were significantly lower compared with the control $(\mathrm{P}<0.05$; Fig. 2$)$. The correlation between GATA-3 and Cx32 protein expression levels in GES-1 cells at $24 \mathrm{~h}$ after $H$. pylori infection was statistically significant ( $\mathrm{r}=-0.7888 ; \mathrm{P}=0.0115)$ (data not shown).

Cx32 mRNA and protein expression levels following GATA-3 siRNA transfection in BGC823 cells. As presented in Fig. 3A, compared with the control group, GATA-3 mRNA expression levels in the GATA-3 siRNA group decreased significantly $(\mathrm{P}<0.05)$, while that in the NC group presented no notable difference, indicating that GATA-3 siRNA specifically and effectively interfered with the expression of GATA-3 mRNA. However, Cx32 mRNA expression levels in the siRNA group were significantly increased $(\mathrm{P}<0.05)$, while that in the NC group had no significant difference $(\mathrm{P}>0.05)$ compared with the control group. The correlation between GATA3 and Cx32 mRNA expression in BGC803 cells in the NC group and GATA-3 siRNA group was statistically significant $(\mathrm{r}=-0.751, \mathrm{P}=0.015 ; \mathrm{r}=-0.880, \mathrm{P}=0.001$, respectively) (data not shown).
As there were no significant differences in GATA-3 and Cx32 mRNA expression levels between the control group and $\mathrm{NC}$ group $(\mathrm{P}>0.05)$, the protein expression levels of GATA-3 and Cx32 in the NC group and siRNA group were detected. The results revealed that the GATA-3 protein expression levels in the siRNA group decreased significantly compared with the NC group $(\mathrm{P}<0.05)$, consistent with the RT-qPCR results, suggesting an effective interference effect. Additionally, Cx32 protein expression levels in the GATA-3 siRNA group were significantly higher compared with the $\mathrm{NC}$ group $(\mathrm{P}<0.05$; Fig. $3 \mathrm{~B}$ and $\mathrm{C})$. The correlation between GATA 3 and Cx32 protein expression in BGC803 cells in the NC group and GATA-3 siRNA group was statistically significant $(\mathrm{r}=-0.867, \mathrm{P}=0.024 ; \mathrm{r}=-0.759, \mathrm{P}=0.018$, respectively) (data not shown).

Changes of GJIC function in GES-1 cells subsequent to $H$. pylori infection. In the control group, the fluorescent dye transferred from the GES-1 cells along the scrape to cells spanning 3-4 rows either side of the scrape, indicating a strong function of GJIC; while in the H.pylori group, the fluorescent dye was mostly limited to a single row of cells next to the scrape, with a limited amount of dye penetrating through to the second row of cells, indicating that the GJIC was weakened or absent, as presented in Fig. 4.

GATA-3 negatively regulates Cx32 by directly binding to its promoter. In the $C \times 32$ promoter sequence -651 to +84 as presented in Fig. 5A, the 6-nucleotide sequences at positions $-623,-514$ and -239 are in accordance with the consensus sequence and may be presumed to be GATA-3 binding sites. 

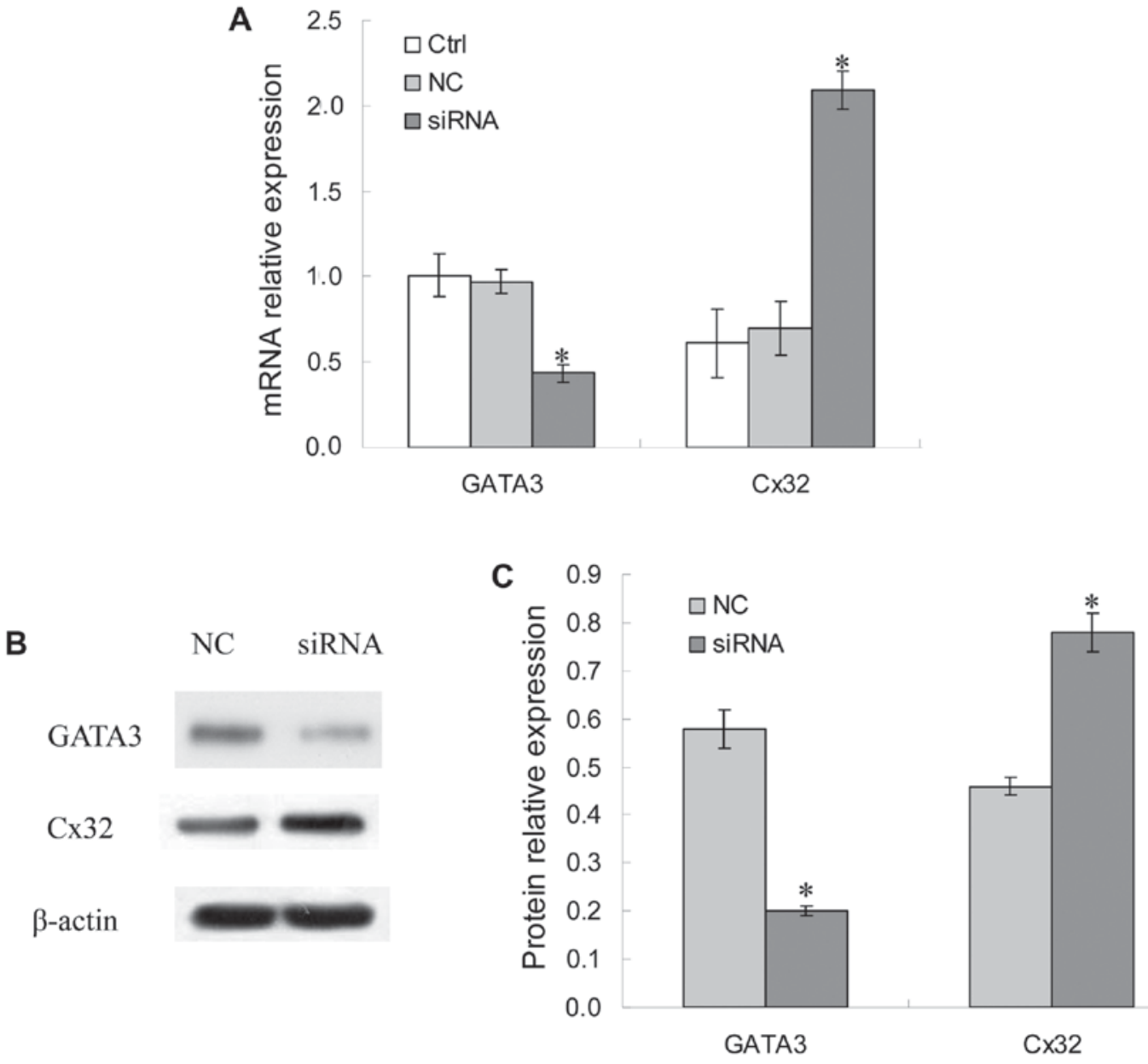

Figure 3. mRNA and protein expression levels of GATA-3 and Cx32 in BGC823 cells in control, NC and GATA-3 siRNA groups. (A) mRNA expression levels in the three groups. (B) Bands of western blot analysis subsequent to electrophoresis in NC and siRNA groups in BGC823 cells. (C) Protein expression levels of GATA3 and Cx32 in BGC823 cells in NC and siRNA groups. "P<0.05 vs. the control. GATA-3, GATA binding protein 3; Cx32, connexin 32; Ctrl, control; $\mathrm{NC}$, negative control; siRNA, small interfering RNA.
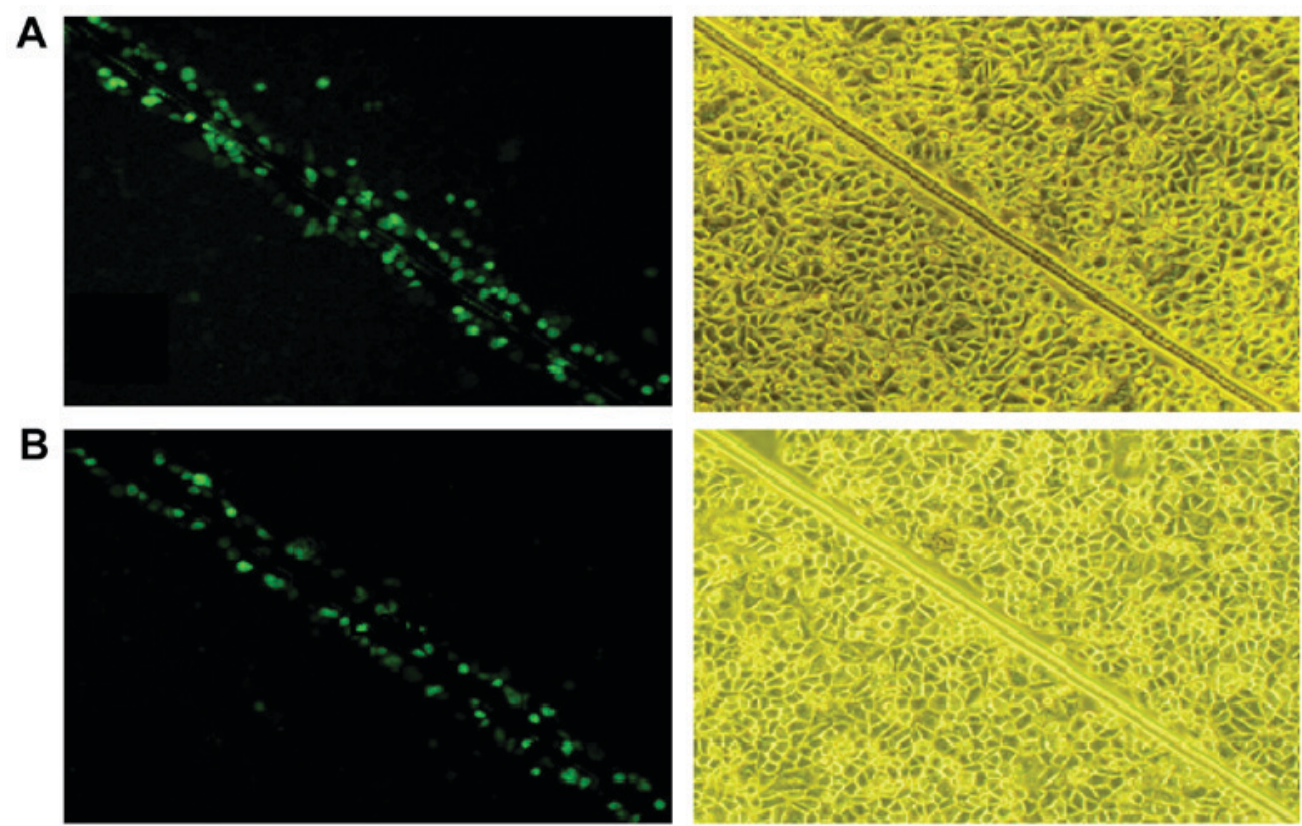

Left

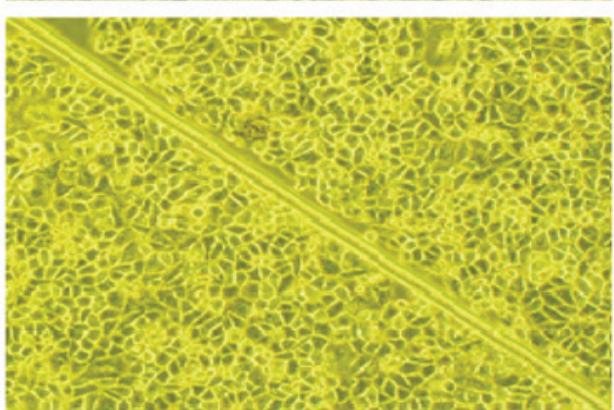

Right

Figure 4. Change of gap junctional intercellular communication function in GES-1 cells cultured for $24 \mathrm{~h}$ following $H$.pylori infection in (A) the control group and (B) the H. pylori group. Left, green fluorescence images; and right, corresponding ordinary light images under light microscope (magnification, $\mathrm{x} 40$ ). H. pylori, Helicobacter pylori. 

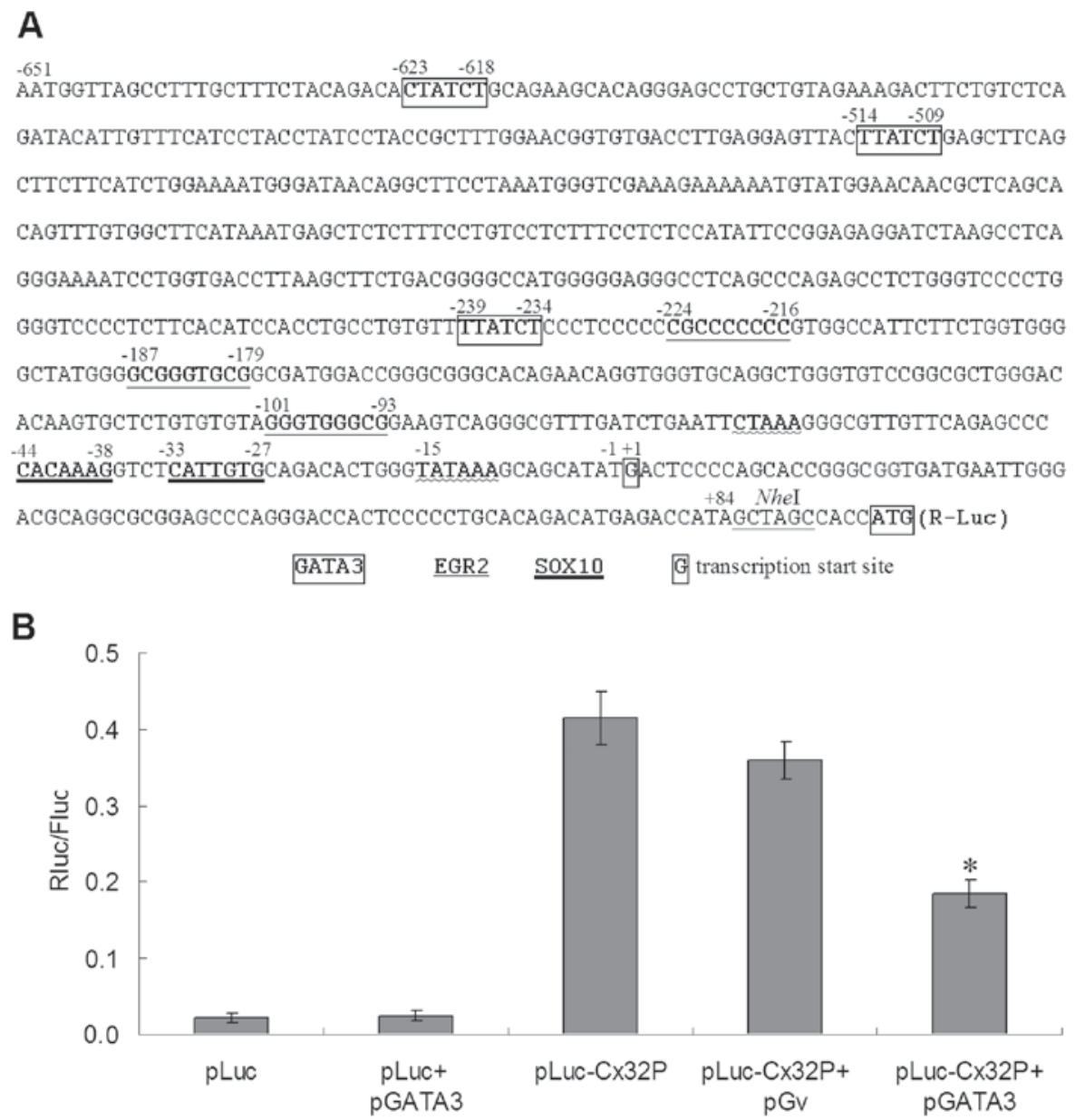

Figure 5. Result of the dual luciferase reporter assay. (A) The $C x 32$ promoter sequence -651 to +84 . Potential transcription factor binding sites: positions $-623,-514$ and -239 for GATA-3; positions $-224,-187$ and -101 for EGR2; and positions -44 and -33 for SOX10. Position -15 for TATA box. Position $+1 \mathrm{G}$ for transcription start site. Boxed ATG for translation start codon. (B) The Rluc/Fluc ratios of different plasmid transfection from the dual luciferase reporter experiment. The mean Rluc/Fluc ratio was 0.022 for pLuc transfection, 0.029 for pLuc+pGATA3, 0.415 for pLuc-Cx32P, 0.359 for $\mathrm{pLuc}-\mathrm{Cx} 32 \mathrm{P}+\mathrm{pGv}$ and 0.185 for pLuc-Cx32P+pGATA3. "P<0.05 vs. pLuc-Cx32P or pLuc-Cx32P+pGv groups. Cx32P, connexin 32 promoter; pLuc, plasmid of dual luciferases; pGv, plasmid GV230 blank vector; pGATA3, plasmid of GATA binding protein 3; Rluc, Renilla luciferase; Fluc, firefly luciferase; EGR2, early growth response 2; SOX10, SRY-Box 10.

The 9-nucleotide sequences at positions -224, -187 and -101 are the consensus sequences of the transcription factor early growth response 2 (EGR2), and the 6-nucleotide sequence at positions -44 and -33 is the consensus sequence of transcription factor SRY-box 10 (SOX10).

In the dual-luciferase reporter experiment, the ratio of Renilla luciferase (Rluc) fluorescence to Firefly luciferase (Fluc) fluorescence $(\mathrm{R} / \mathrm{F}$ ratio) reflected the activity of inserted promoter sequence. As presented in Fig. 5B, the mean R/F ratio of pLuc transfection was 0.022 , and that of pLuc+pGATA3 was 0.029 , indicating that GATA 3 overexpression has no effect on the reporter plasmid without a $C \times 32$ promoter. The mean $\mathrm{R} / \mathrm{F}$ ratio of pLuc-Cx32P transfection was 0.415 , and that of pLuc-Cx32P+pGv was 0.359 , suggesting that $C \times 32$ promoter is activated in GES-1 cells, while co-transfection with another plasmid may decrease the activity of the promoter, but the difference in promoter activity with or without another plasmid was not significant $(\mathrm{P}>0.05)$. The mean $\mathrm{R} / \mathrm{F}$ ratio of pLuc-Cx32P+pGATA3 transfection was 0.185 , which is significantly different compared with pLuc-Cx32P and pLuc-Cx32P+pGv $(\mathrm{P}<0.05)$, indicating that GATA-3 inhibits expression activity by direct binding to the promoter.

\section{Discussion}

In the present study, the expression levels of GATA-3 mRNA and protein in $H$. pylori-infected GES-1 cells increased with increasing incubation time, and also in the gerbil gastric antrum tissues following $H$. pylori infection. Whilst the mechanism of how $H$. pylori bacteria may activate the expression of GATA-3 remains unclear, the mechanism underlying GATA-3 overexpression promoting carcinogenesis was investigated in the present study. H. pylori may interact with the epithelial cells and infiltrate natural killer (NK) cells in the gastrointestinal tract. According to Lindgren et al (17), co-culture with H.pylori lysate caused the GATA-3 overexpression, which may inhibit the transforming growth factor- $\beta$ expression, thereby inhibiting NK cell activity, resulting in the decreased interferon- $\gamma$ secretion by NK cells, thus increasing the risk of carcinogenesis in gastric epithelial cells. This is the potential mechanism by which the high expression of GATA-3 participates in the regulation of tumor immunity resulting in tumor immune escape.

In the present study, once the gastric epithelial cells were infected with $H$.pylori, the $\mathrm{Cx} 32$ expression at the transcriptional and protein levels were decreased with increasing incubation 
time, and GJIC function was inhibited. This is consistent with previous results in gastric precancerous lesions (10). The loss of GJIC function in tumor cells has been associated with the downregulated tissue-specific expression of connexin genes (18). Cx32 is the principal protein constituting the gap junction in gastric epithelial cells (19). It was additionally revealed that in gastric carcinogenesis, the expression of Cx32 was decreased in chronic non-atrophic gastritis, gastric precancerous lesions and GC, with the lowest expression in GC (10). In the present study, it was hypothesized that GATA-3 may downregulate the expression of $\mathrm{Cx} 32$, which then promotes the tumor incidence by decreasing the intercellular connection and communication.

In the present study, the association between the expression of GATA-3 and expression of Cx32 was examined. By siRNA transfection, the expression of GATA-3 was negatively associated with Cx32 expression in GES-1 cells. The fluorescent dyes of the $H$.pylori-infection group were mostly limited to the single cell line surrounding the scratch, with only a limited amount of dye penetrating through to the second cell line, indicating that the GJIC function was substantially reduced or absent, while in the control group, the fluorescence dye transferred to the neighboring 3-4 column cells, demonstrating a stronger GJIC function. Using a dual luciferase reporter assay, GATA-3 inhibited the expression of the luciferase reporter downstream to the Cx32 promoter, suggesting that GATA-3 inhibits the expression of $\mathrm{Cx} 32$ by binding to the $\mathrm{Cx} 32$ promoter sites. These experiments revealed that GATA-3 inhibits the Cx32 expression activity by direct binding to the $\mathrm{Cx} 32$ promoter.

Cx32 is positively regulated by the transcription factors SOX10 and EGR2 functioning at the Cx32 promoter -44 to -38 and -33 to -27 sites, and -101 to $-93,-187$ to -179 and -224 to -216 sites $(20,21)$. In the present study, the potential binding sites of GATA3 were identified to be -239 to $-234,-514$ to -509 and -623 to -618 , all upstream of the SOX10 and EGR2 binding sites. As a novel high-mobility group-box-containing tumor suppressor, SOX10 may additionally inhibit the growth and metastasis of digestive tract cancer types by suppressing the Wnt/ $\beta$-catenin pathway (22). The EGR2 signaling pathway also attenuated the carcinogenesis of GC (23). These indicate that in the Cx32 promoter, SOX10 and EGR2 function as activating factors whilst GATA3 functions as an inhibiting factor, which is consistent with their general roles in GC development.

Certain relevant questions should be mentioned. Firstly, whether the expression of GATA-3 in different tumor types or tumor tissues is upregulated or downregulated varies between studies (24). The result of the present study indicates that GATA-3 is highly expressed in the GC cell line BGC823, which is consistent with markedly higher GATA-3 expression in GC tissues (13). Similar results have been demonstrated in pancreatic cancer and pancreatic carcinoma cell lines (25). GATA3 is expressed in a wide variety of benign and malignant cutaneous epithelial neoplasms (26). But in breast cancer, GATA3 expression has been associated with estrogen receptor positive (ER+/luminal) phenotypes, accounting for $2 / 3$ of all breast cancer cases, while loss of GATA3 expression is associated with ER-, less differentiated, invasive breast cancer (24). As a mechanism, progestin-activated progesterone receptor (PR) reduces GATA3 expression through regulation at the transcriptional and post-translational levels, promoting tumor growth (27). Therefore, in order to provide novel therapeutic targets, further investigation of GATA3-associated pathways will be necessary to further understanding of different cancer incidence and dissemination.

Secondly, the association between upregulated GATA-3 and carcinogenesis is multifaceted. GATA-3 is additionally a specific transcription factor for Th2 type cytokines, its abnormally high expression not only is able to promote the development of $\mathrm{T}$ helper (Th) 2 cells, but may also inhibit cell differentiation in the Thl direction, resulting in a Thl/Th2 type cytokine imbalance to exhibit immunosuppression (28). Tumor formation is closely associated with the immune escape of tumor antigens, and the body'simmune tolerance and immune suppression promote tumor growth and metastasis (29).

Finally, the results suggest that $H$. pylori infection causes upregulation of GATA-3 and decreases the expression of Cx32 and GJIC function. It is possible to intervene with this regulating course to improve the intercellular communication, benefiting the prevention and therapy of gastric cancer. Clinical and experimental studies in vitro confirmed that $H$. pylori may significantly reduce GJIC function in gastric epithelial cells $(11,12)$. One previous study demonstrated that the eradication of $H$. pylori may increase $\mathrm{C} \times 32$ expression in precancerous lesions, thereby maintaining GJIC function (10). The expression of Cx32 in BGC823 cells was upregulated by GATA-3 siRNA transfection in the present study, yet the application of GATA-3 siRNA in the gastric antrum tissues remains questionable. Cx32 may also inhibit cell proliferation by cell cycle arresting effects and cell cycle regulatory proteins, and thus serves an important function in the inhibition of GC development (30). Therefore, enhancement of Cx32 expression by demethylation of the $C x 32$ gene promoter may also provide ways to prevent gastric carcinogenesis (31). Together with the consistent methods and results in a previous study on $\mathrm{Cx} 43$ expression in gastric carcinogenesis (32), potential methods in enhancing GJIC function may be effective in preventing and treating GC.

To conclude, the present study revealed that there is a negative association between GATA-3 and Cx32 expression, suggesting that $H$. pylori infection may upregulate GATA-3 expression, which negatively regulates the expression of its downstream target gene $\mathrm{Cx} 32$, resulting in GJIC dysfunction, therefore serving an important function in the incidence and development of GC. The detailed mechanisms, general meaning and therapeutic application need to be further explored.

\section{Acknowledgements}

Not applicable.

\section{Funding}

The present study is supported by the National Natural Science Foundation of China (grant nos. 81172301 and 81570509), National Innovative Training Program of China (grant no. 201310533059) and the Science and Technology Project of Changsha (grant no. k1406048-31).

\section{Availability of data and materials}

All data generated or analyzed during this study are included in this published article. 


\section{Authors' contributions}

LH arranged the experiments, analyzed the data and wrote the manuscript. CX designed the experiments and revised the manuscript. DC, YG, XL, KC, TH and YQ performed the experiments. $\mathrm{LZ}$ analyzed the data.

\section{Ethics approval and consent to participate}

This study was approved by the Ethics Committee of Third Xiangya Hospital of Central South University, China. The patients provided their written informed consent to participate in this study.

\section{Consent for publication}

The patients provided their written informed consent for their data to be published.

\section{Competing interests}

The authors declare that they have no competing interests.

\section{References}

1. Chen W, Zheng R, Baade PD, Zhang S, Zeng H, Bray F, Jemal A, Yu XQ and He J: Cancer statistics in China, 2015. CA Cancer J Clin 66: 115-132, 2016.

2. Grabsch HI and Tan P: Gastric cancer pathology and underlying molecular mechanisms. Dig Surg 30: 150-158, 2013.

3. Ahn HJ and Lee DS: Helicobacter pylori in gastric carcinogenesis. World J Gastrointest Oncol 7: 455-465, 2015.

4. Kim SS, Ruiz VE, Carroll JD and Moss SF: Helicobacter pylori in the pathogenesis of gastric cancer and gastric lymphoma. Cancer Lett 305: 228-238, 2011.

5. Matsubara S, Takasu S, Tsukamoto T, Mutoh M, Masuda S, Sugimura T, Wakabayashi K and Totsuka Y: Induction of glandular stomach cancers in Helicobacter pylori-infected Mongolian gerbils by 1-nitrosoindole-3-acetonitrile. Int J Cancer 130: 259-266, 2012.

6. Zheng Q, Chen XY, Shi Y and Xiao SD: Development of gastric adenocarcinoma in Mongolian gerbils after long-term infection with Helicobacter pylori. J Gastroenterol Hepatol 9: 1192-1198, 2004.

7. Mesnil M, Crespin S, Avanzo JL and Zaidan-Dagli ML: Defective gap junctional intercellular communication in the carcinogenic process. Biochim Biophys Acta 1719: 125-145, 2005.

8. Wu J, Zhou HF, Wang CH, Zhang B, Liu D, Wang W and Sui GJ: Decreased expression of $\mathrm{Cx} 32$ and $\mathrm{Cx} 43$ and their function of gap junction intercellular communication in gastric cancer. Zhonghua Zhong Liu Za Zhi 29: 742-747, 2007 (In Chinese).

9. Xu CX, Qi YM, Yang WB, Wang F, Zhou JD and Shen SR: Effect of $\mathrm{CagA}^{+}$helicobacter pylori strain on the expression of connexin 43 and cell proliferation in BGC2823 cells. Zhong Nan Da Xue Xue Bao Yi Xue Ban 32: 288-294, 2007 (In Chinese).

10. Xu CX, Jia Y, Yang WB, Wang F and Shen SR: Relationship between Helicobacter pylori infection and expression of connexin (Cx) 32 and $\mathrm{Cx} 43$ genes in gastric cancer and gastric precancerous lesions. Zhonghua Yi Xue Za Zhi 88: 1523-1527, 2008 (In Chinese).

11. Jia Y, Xu CX and Yang WB: Expressions of connexin 32 and connexin 43 in patients with gastric precancerous lesion after eradication of Helicobacter pylori. Zhong Nan Da Xue Xue Bao Yi Xue Ban 33: 628-633, 2008 (In Chinese).

12. Xu CX, Jia Y, Yang WB, Zou HF, Wang F and Shen SR: Helicobacter pylori infection and changes of cell gap junction of gastric epithelial cells in patients with gastric cancer and precancerous lesion. Zhong Nan Da Xue Xue Bao Yi Xue Ban 33: 338-343, 2008 (In Chinese).

13. Hu TZ, Huang LH, Xu CX, Liu XM, Wang Y, Xiao J, Zhou L, Luo L and Jiang XX: Expressional profiles of transcription factors in the progression of Helicobacter pylori-associated gastric carcinoma based on protein/DNA array analysis. Med Oncol 32: 265, 2015.
14. Livak KJ and Schmittgen TD: Analysis of relative gene expression data using real-time quantitative PCR and the 2(-Delta Delta C(T)) method. Methods 25: 402-408, 2001.

15. Sadat MA, Kumatori A, Suzuki S, Yamaguchi Y, Tsuji Y and Nakamura M: GATA-3 represses gp91phox gene expression in eosinophil-committed HL-60-C15 cells. FEBS Lett 436: 390-394, 1998.

16. Shield PW, Papadimos DJ and Walsh MD: GATA3: A promising marker for metastatic breast carcinoma in serous effusion specimens. Cancer Cytopathol 122: 307-312, 2014.

17. Lindgren $\AA$, Yun CH, Sjöling Å, Berggren C, Sun JB, Jonsson E, Holmgren J, Svennerholm AM and Lundin SB: Impaired IFN- $\gamma$ production after stimulation with bacterial components by natural killer cells from gastric cancer patients. Exp Cell Res 317: 849-858, 2011.

18. Saito T, Nishimura M, Kudo R and Yamasaki H: Suppressed gap junctional intercellular communication in carcinogenesis of endometrium. Int J Cancer 93: 317-323, 2001.

19. Uchida Y, Matsuda K, Sasahara K, Kawabata H and Nishioka M: Immunohistochemistry of gap junctions in normal and diseased gastric mucosa of humans. Gastroenterology 109: 1492-1496, 1995.

20. Bondurand N, Girard M, Pingault V, Lemort N, Dubourg O and Goossens M: Human connexin 32, a gap junction protein altered in the X-linked form of Charcot-Marie-Tooth disease, is directly regulated by the transcription factor SOX10. Hum Mol Genet 10: 2783-2795, 2001.

21. Houlden H, Girard M, Cockerell C, Ingram D, Wood NW, Goossens M, Walker RW and Reilly MM: Connexin 32 promoter P2 mutations: A mechanism of peripheral nerve dysfunction. Ann Neurol 56: 730-734, 2004.

22. Tong X, Li L, Li X, Heng L, Zhong L, Su X, Rong R, Hu S, Liu W, Jia B, et al: SOX10, a novel HMG-box-containing tumor suppressor, inhibits growth and metastasis of digestive cancers by suppressing the $\mathrm{Wnt} / \beta$-catenin pathway. Oncotarget 5 : 10571-10583, 2014

23. Li X, Zhang Z, Yu M, Li L, Du G, Xiao W and Yang H: Involvement of miR-20a in promoting gastric cancer progression by targeting early growth response 2 (EGR2). Int J Mol Sci 14: 16226-16239, 2013.

24. Cohen H, Ben-Hamo R, Gidoni M, Yitzhaki I, Kozol R, Zilberberg A and Efroni S: Shift in GATA3 functions, and GATA3 mutations, control progression and clinical presentation in breast cancer. Breast Cancer Res 16: 464, 2014.

25. Gulbinas A, Berberat PO, Dambrauskas Z, Giese T, Giese N, Autschbach F, Kleeff J, Meuer S, Büchler MW and Friess H: Aberrant GATA-3 expression in human pancreatic cancer. J Histochem Cytochem 54: 161-169, 2006.

26. Mertens RB, de Peralta-Venturina MN, Balzer BL and Frishberg DP: GATA3 Expression in normal skin and in benign and malignant epidermal and cutaneous adnexal neoplasms. Am J Dermatopathol 37: 885-891, 2015.

27. Izzo F, Mercogliano F, Venturutti L, Tkach M, Inurrigarro G, Schillaci R, Cerchietti L, Elizalde PV and Proietti CJ: Progesterone receptor activation downregulates GATA3 by transcriptional repression and increased protein turnover promoting breast tumor growth. Breast Cancer Res 16: 491, 2014.

28. Chou J, Provot S and Werb Z: GATA-3 in development and cancer differentiation: Cells GATA Have It! J Cell Physiol 222: 42-49, 2010.

29. Tashireva LA, Perelmuter VM, Manskikh VN, Denisov EV, Savelieva OE, Kaygorodova EV and Zavyalova MV: Types of immune-inflammatory responses as a reflection of cell-cell interactions under conditions of tissue regeneration and tumor growth. Biochemistry (Mosc) 82: 542-555, 2017.

30. Jee H, Lee SH, Park JW, Lee BR, Nam KT and Kim DY: Connexin32 inhibits gastric carcinogenesis through cell cycle arrest and altered expression of p21Cip1 and p27Kip1. BMB Rep 46: 25-30, 2013.

31. Wang Y, Huang LH, Xu CX, Xiao J, Zhou L, Cao D, Liu XM and Qi Y: Cx32 and Cx43 promoter methylation in Helicobacter pylori-associated gastric tumorigenesis. World J Gastroenterol 20: 11770-11779, 2014.

32. Liu X, Cao K, Xu C, Hu T, Zhou L, Cao D, Xiao J, Luo L, Guo Y and Qi Y: GATA-3 augmentation down-regulates Connexin43 in Helicobacter pylori associated gastric carcinogenesis. Cancer Biol Ther 16: 987-996, 2015.

This work is licensed under a Creative Commons Attribution-NonCommercial-NoDerivatives 4.0 International (CC BY-NC-ND 4.0) License. 